\title{
Frontières
}

\section{Pour que la vie nous soit bonne, jusqu’à notre mort}

\section{Diane Laflamme}

Volume 20, numéro 1, automne 2007

La « bonne mort »

URI : https://id.erudit.org/iderudit/017939ar

DOI : https://doi.org/10.7202/017939ar

Aller au sommaire du numéro

Éditeur(s)

Université du Québec à Montréal

ISSN

1180-3479 (imprimé)

1916-0976 (numérique)

Découvrir la revue

Citer ce document

Laflamme, D. (2007). Pour que la vie nous soit bonne, jusqu'à notre mort.

Frontières, 20(1), 3-6. https://doi.org/10.7202/017939ar d'utilisation que vous pouvez consulter en ligne.

https://apropos.erudit.org/fr/usagers/politique-dutilisation/ 


$\begin{array}{llllllllll}\text { E } & \text { D } & \text { I } & \text { T } & 0 & \text { R } & \text { I } & \text { A } & \text { L }\end{array}$
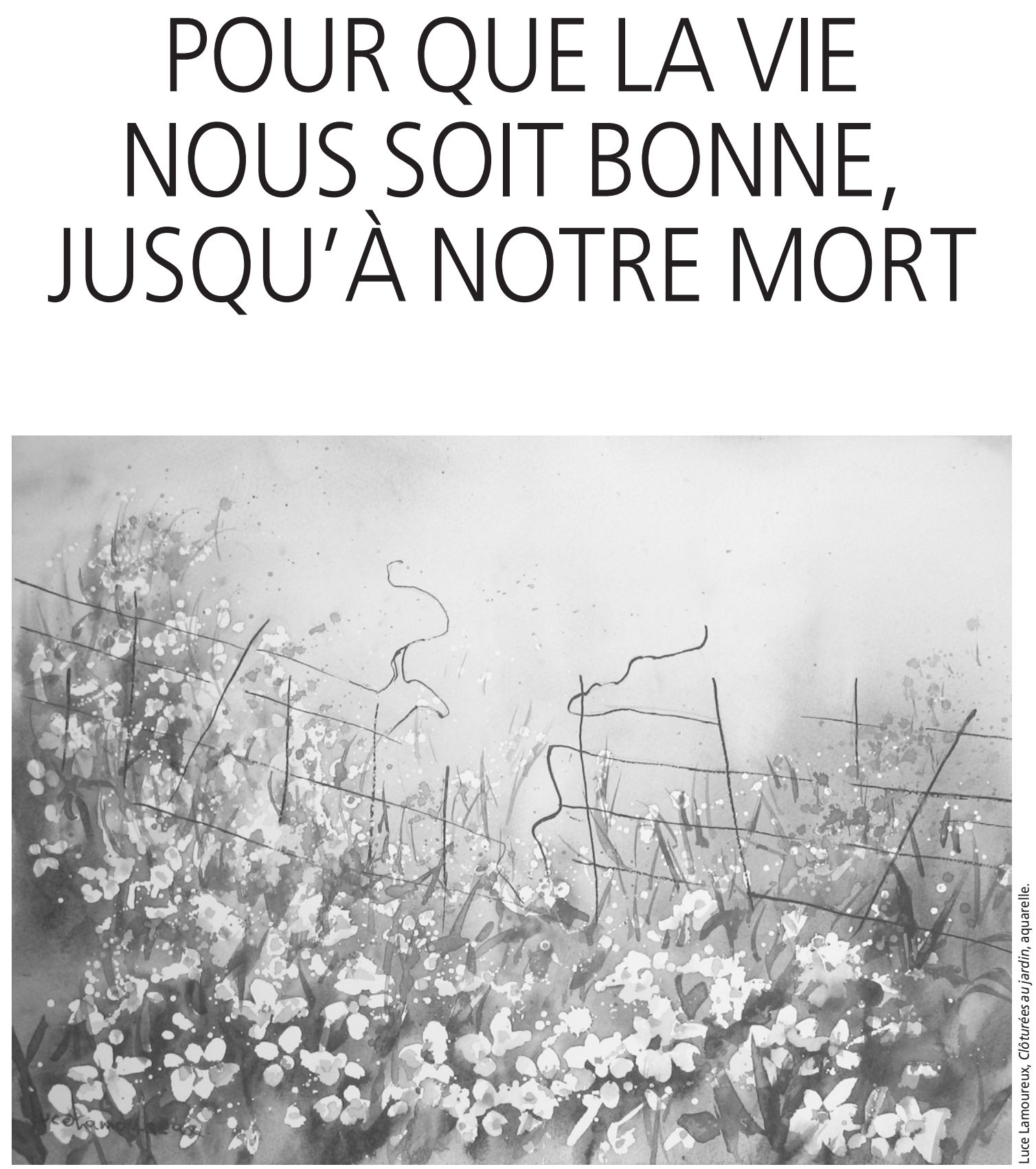

Diane Laflamme, Ph. D., rédactrice en chef.

Nous voulons tout: la vie bonne maintenant, et la bonne mort quand l'heure viendra! Mais nous craignons de finir par perdre ce qui est bon pour nous; nous avons surtout peur de souffrir de ces pertes au plus vif de notre chair, lieu irréductible de ce qui, en nous, est capable de goûter tout le «bon» qu'il y a dans notre vie.

Soyons réalistes: nous allons tout perdre! Pour nous en convaincre, il suffit d'une visite dans un hôpital ou dans un centre de soins prolongés. Les consommateurs sophistiqués que nous sommes peuvent déjà y contempler les quelques biens et meubles auxquels ils auront accès, les quelques mètres carrés sans confort véritable qu'ils habiteront, comme tout le monde.

Mais n'en restons pas aux pertes en biens et meubles du consommateur; il y a aussi les grandes valeurs du citoyen, comme la liberté et la dignité. Le citoyen québécois, par exemple, est libre de refuser les traitements médicaux qui lui sont proposés ${ }^{1}$ et le mot dignité est mentionné deux fois dans la Charte des droits et libertés de la personne du Québec: dans le préambule et à l'article 4 où il est écrit que «[t]oute personne a droit à la sauvegarde de sa dignité $[\ldots] »$. 
Quand nous envisageons ce que sera la vie bonne pour nous lorsque nous serons gravement malades ou très âgés, nous avons pourtant des raisons d'avoir peur que notre liberté et notre dignité soient compromises. Que reste-t-il de cette liberté de choisir et de cette dignité quand le corps et l'intellect deviennent le champ de bataille de processus biologiques qui se détraquent et d'interventions chimiques qui contre-attaquent? La liberté d'action, ce sont d'abord nos soignants qui l'auront. Mais le salaire horaire et l'agenda de travail de ceux qui sont bardés de diplômes limiteront le temps qu'ils pourront librement passer à notre chevet, alors que le salaire horaire et l'agenda de travail des autres, les préposés aux bénéficiaires surtout, limiteront leur capacité d'ajouter librement à leur liste de tâches le souci de respecter notre dignité et notre liberté de choisir, par exemple, quand ou comment nous voulons être alimentés ou lavés... Nos aidants naturels feront de leur mieux pour respecter notre liberté et notre dignité, leur propre liberté étant rudement mise à l'épreuve par le défi que nos défaillances leur imposeront ${ }^{2}$.

Une première ligne de défense pour préserver notre vie bonne, notre liberté et notre dignité jusqu'à notre mort, ce sont les soins palliatifs. Déjà beaucoup de personnes, des professionnels et des bénévoles, se dévouent pour mettre toutes les ressources regroupées sous l'appellation de soins palliatifs à la disposition des malades en phase terminale. Nos administrations publiques, dans les textes officiels, se déclarent en faveur d'un investissement majeur dans les soins palliatifs, et il faut s'en féliciter. Mais, les mesures concrètes requises pour que ce vœu se réalise ne progressent qu'à pas de tortue: il faut s'en inquiéter ${ }^{3}$.

Une seconde ligne de défense, déjà fermement en place au Québec et au Canada, c'est le refus de l'acharnement thérapeutique, de l'obstination déraisonnable. Car nous avons peur de devenir des «légumes », dans les serres vitrées d'une technologie médicale capable de prouesses inimaginables et qui n'aurait pas de compte à nous rendre si elle fonctionnait uniquement selon une logique institutionnalisée entre pairs. C'est là le pire des scénarios. Entre ce cauchemar et la «bonne mort» que nous espérons, il y a toute une échelle dans le niveau et l'agressivité des traitements qui nous seront offerts. Le citoyen québécois peut formuler des «directives préalables», communément appelées «testament biologique» (Dunsmuir et Tiedemann, p. 11-12). Afin de pouvoir faire connaître aux personnes appropriées notre volonté quant à l'intensité des soins que nous recevrons et, surtout, afin de pouvoir continuer de faire reconnaître notre volonté sur une longue période, alors même que nos moyens pour nous exprimer s'amenuiseront peu à peu, nous devrons cependant pouvoir compter sur des équipes soignantes qui auront mis en place les mécanismes requis pour que notre voix affaiblie par la maladie terminale ou le grand âge se fasse encore entendre dans les dédales de l'institution ${ }^{4}$.

La mort assistée, qu'elle prenne la forme d'une euthanasie volontaire ou d'une aide au suicide ${ }^{5}$, constitue-t-elle une troisième ligne de défense pour préserver notre vie bonne, notre liberté et notre dignité jusqu'à notre mort? Pour le citoyen du Québec $^{6}$, la réponse est non. La personne qui accepte d'en aider une autre à mourir pourra être citée devant les tribunaux, même si elle agissait à la demande expresse d'un être aimé qui voyait là sa «bonne mort». La tragédie vécue le 24 septembre 2004 par une mère qui a aidé son fils de 36 ans, atteint de sclérose en plaques, à se suicider nous l'a rappelé douloureusement. Les journaux ont abondamment décrit la tragédie, sans omettre le sac de plastique que la mère a dû maintenir autour de la tête de son fils après qu'il eut avalé un cockail de médicaments, le tout selon des directives glanées sur Internet ${ }^{7}$.

Ils étaient tous deux des soignants: la mère était une infirmière auxiliaire à la retraite, et le fils, auteur dramatique, avait aussi travaillé comme préposé aux bénéficiaires au Centre de soins prolongés Saint-Charles-Borromée, à Montréal. La sentence du juge de la Cour supérieure du Québec, rendue le 27 janvier 2006, a été de trois ans de probation pour Marielle Houle - alors au début de la soixantaine - au lieu des quatorze années de prison qui auraient pu être imposées. On peut rappeler ici, pour comparer la réalité et la fiction, ce que peut se permettre le personnage de Rémy dans le film de Denys Arcand, Les invasions barbares: Rémy quitte l'hôpital où il reçoit des soins pour une maladie terminale et se rend avec son fils et ses amis dans un lieu agréable, à la campagne, où il a accès à l'aide nécessaire pour mettre fin paisiblement à ses jours en s'injectant lui-même une substance létale. Ce film véhiculait une image puissante de ce que pourrait être une «bonne mort» - à la brunante près d'un lac des Laurentides -, mais le cinéaste québécois ne nous dit pas si celles et ceux qui ont aidé Rémy à mourir ont ensuite été poursuivis par la justice comme ce fut le cas pour Marielle Houle.

Les trois lignes de défense que nous venons d'évoquer confirment que nous avons irrémédiablement besoin d'autrui pour vivre dans la liberté et dans la dignité jusqu'à notre mort. Nous n'y arriverons pas tout seuls. Nous avons constamment besoin d'autrui : à la fois pour que la vie nous soit bonne et pour que la mort nous soit bonne. Nous avons besoin d'autrui pour qu'il nous fournisse des soins palliatifs, pour qu'il nous écoute quand nous lui demanderons de s'abstenir de nous donner des traitements dont nous ne voulons plus et, dans les pays où les lois le permettent - et comme le propose maintenant au Québec l'association nouvellement créée pour le droit de mourir dans la dignité ${ }^{8}$-, pour qu'il accepte de nous aider à mettre fin à nos jours si nous le lui demandons afin d'éviter une déchéance ou une souffrance que nous jugeons insupportables.

À qui nous adresserons-nous pour obtenir l'aide qui nous permettra de vivre dans la dignité jusqu'au moment de notre mort, que ce soit sous la forme des soins palliatifs, du respect de nos volontés quant à l'intensité des soins ou, éventuellement, de la mort assistée ? Qui nous aidera à vivre notre version de la «bonne mort», dans la liberté et la dignité? Une mère, des amis $^{9}$, des soignants?

Avoir besoin d'autrui comme soignant, c'est une expérience déjà documentée dans les textes de Platon. Ce sont d'ailleurs les anciens Grecs, plus précisément Aristote, qui nous ont légué les notions de «vie bonne » et de liberté du citoyen de la cité. Mais, les citoyens de la Grèce antique avaient des esclaves, nous le savons, et les concepts de vie bonne et de liberté n'avaient pas été pensés pour eux. Ces esclaves avaient des médecins, eux aussi esclaves. Lisons comment Platon décrit le lien entre le médecin et son patient chez les esclaves:

Réfléchis-tu en outre à ceci que, dans les cités, les malades étant de condition libre ou esclaves, ce sont le plus souvent, peu s'en faut, les esclaves qui sont les médecins des esclaves, soit qu'ils courent à droite et à gauche, soit qu'ils restent en permanence dans l'officine; et que, de ces sortes de médecins, aucun ne donne à chacun des serviteurs qu'il soigne aucune explication sur la maladie dont souffre celui-ci, pas plus qu'il ne consent à en recevoir de lui. Mais, après lui avoir fait l'ordonnance que lui dicte sa routine, faisant l'homme qui sait son affaire sur le bout du doigt, avec une arrogance de tyran, il court d'un bond à un autre serviteur malade; [...] (Platon, 1950, p. 769).

Platon ne serait probablement pas dépaysé s'il visitait les salles d'urgence de nos grands hôpitaux modernes... Voyons maintenant comment il décrit la relation entre le citoyen libre et son médecin, lui aussi citoyen libre:

Or, c'est le médecin de condition libre qui, en général, soigne et traite les maladies des gens appartenant à la même 
condition; après avoir procédé à un examen du mal depuis son début et, à la fois, selon ce qu'exige la nature d'un tel examen, entrant en conversation, tant avec le patient lui-même qu'avec ses amis, ainsi, en même temps que du malade il apprend personnellement quelque chose, en même temps aussi, dans toute la mesure où il le peut, il instruit à son tour celui qui est en mauvaise santé; bien plus, il n'aura rien prescrit qu'il n'ait auparavant de quelque façon gagné sa confiance (Platon, 1950, p. 769; notre soulignement).

Ce qui caractérise la relation entre les personnes libres que décrit Platon: le médecin, le patient et ses amis, c'est la conversation à laquelle ils acceptent de prendre part. Une conversation qui permet aux parties d'apprendre l'une de l'autre et d'établir un lien de confiance ${ }^{10}$. Car avoir besoin d'autrui, c'est là un risque énorme! Autrui est capable de toutes les métamorphoses: de toutes les grandeurs et de toutes les bassesses. Autrui peut nous aider conformément à ce que nous lui demandons, autrui peut nous aider en faisant plutôt ce qu'il croit bon pour nous, autrui peut à tout moment profiter de notre faiblesse et l'exploiter à son profit. L'intervention d'autrui sera-t-elle une bénédiction ou une plaie?

Avoir besoin d'autrui, c'est une expérience qui se vit à deux niveaux: autrui proche, celui que l'on recherche dans l'amitié, et autrui lointain, celui que l'on rencontre dans les institutions. Avoir besoin d'autrui, ce n'est pas seulement compter sur ses proches, ses amis ; c'est aussi compter sur le soutien de la collectivité que nous habitons et que nous contribuons à bâtir en payant nos taxes et en participant à la vie politique.

Quand viendra l'heure, il nous faudra bien accepter de faire confiance à autrui dans l'espoir de «bien» mourir, et sa propre définition de la liberté et de la dignité aura probablement plus de poids que la nôtre pour orienter les choix qu'il fera dans le but de nous aider. Nous aurions avantage à intensifier et à consolider dès maintenant notre lien de confiance avec autrui, au plan personnel d'abord mais aussi à l'échelle de notre collectivité. Cela fait partie de notre effort de citoyen pour construire une société où nous exerçons ce que nous appelons, avec le philosophe Paul Ricoeur, notre visée éthique, c'est-à-dire : la visée de la vie bonne, avec et pour autrui, dans des institutions justes (1995, p. 202-236).

Ces «institutions justes» ne se mettront pas en place d'ellesmêmes. Il nous faudra mettre l'épaule à la roue pour éviter des drames comme celui qu'a vécu Marielle Houle dans l'appartement de son fils. L'Assemblée nationale du Québec - et c'est aussi le cas pour les Parlements des autres provinces - n'a pas jusqu'ici légiféré spécifiquement sur la fin de vie. Lorsqu'ils sont confrontés à des questions difficiles qui se posent en fin de vie - par exemple jusqu'où on peut aller pour demander ou fournir une aide médicale visant à permettre à une personne de vivre ce qu'elle considère une «bonne » mort -, les usagers du système de santé québécois, leurs proches et les soignants doivent se référer à des repères multiples, énoncés dans divers textes, de portée différente, entre autres : le Code criminel canadien, le Code civil du Québec, les Chartes québécoise et canadienne des droits et libertés de la personne, les jurisprudences pertinentes, le Code de déontologie des médecins, le Code de déontologie des infirmières et - c'est parfois le cas depuis peu - les recommandations des Comités d'éthique clinique des établissements du réseau de la santé (Dunsmuir et Tiedemann, 2006, p. 4; Observatoire de l'administration publique de l'ENAP, 2005, p. A-15).

Nous pouvons choisir de repenser l'encadrement législatif dont notre génération a hérité. La députée au Parlement fédéral, Francine Lalonde, y a invité concrètement les Canadiens en présentant en 2005 le projet de loi C-407, Loi modifiant le Code criminel (droit de mourir dignement) ${ }^{11}$. Que l'on soit pour ou contre le contenu de ce projet de loi, il importe de contribuer au débat public sur ce sujet, car il en va de notre accès tant à la vie bonne qu'à la «bonne mort». Cet accès doit se déployer dans des «institutions justes», mises en place par des citoyens qui se donnent la peine de s'atteler à des tâches même difficiles. Notre visée, en tant que société, doit constamment être repensée, réajustée pour tenir compte des connaissances et des ressources additionnelles dont nous disposons, génération après génération, pour rendre et la vie et la mort «bonnes» pour nous, pour nos parents et pour la génération qui va nous suivre.

L'Association canadienne de soins palliatifs (ACSP) a approuvé, le 22 novembre 2006, un Document de réflexion sur l'euthanasie, le suicide assisté et les soins de fin de vie de qualité $^{12}$. Ce document se termine par des recommandations et un Projet de déclaration qui, écrit l'ACSP, «tente de saisir à la fois [la] préoccupation fondamentale à l'égard des questions entourant l'euthanasie et le suicide médicalement assisté, ainsi que [le] respect pour les personnes qui ne partagent pas cette opinion» (p. 4).

Ce Projet de déclaration s'énonce comme suit:

L'Association canadienne de soins palliatifs s'engage

à améliorer la qualité de la vie et de la mort pour les

personnes mourantes et leurs familles, et elle considère

que l'euthanasie et le suicide médicalement assisté ne font

pas partie de soins de fin de vie de qualité.

La fin de la vie sera toujours associée à une certaine souffrance. De bons soins de fin de vie peuvent atténuer une partie de cette souffrance en allégeant le fardeau pour les familles et en les aidant à prendre en charge la douleur et les symptômes. Pour beaucoup de gens, des soins palliatifs de grande qualité sont une meilleure option que l'euthanasie ou le suicide médicalement assisté.

Toutefois, malgré l'accès à des soins palliatifs de grande qualité, un très petit nombre de Canadiens pourraient tout de même vouloir contrôler leur propre mort. En tant que praticiens en soins palliatifs, nous devons respecter leur droit à ce choix et ne pas les abandonner. Nous continuerons à prodiguer les mêmes soins empreints de compassion à ces personnes et à leurs familles, mais nous avons également le droit de ne pas participer ou de ne pas assister tout effort visant à hâter volontairement la mort (p. 4-5).

L'Association canadienne de soins palliatifs déclare ouvertement qu'elle «aimerait savoir ce que pensent ses membres à ce sujet et encourager un débat au sein de la communauté des soins palliatifs» (p. 6). Le Réseau de soins palliatifs du Québec s'est déjà donné, dans son Plan stratégique 2007-2010, l'objectif d'«enrichir le débat public sur l'euthanasie et le suicide assisté, en mettant en évidence le point de vue des soins palliatifs $»^{13}$.

Nous avons probablement beaucoup à apprendre sur le sujet complexe qu'est la «bonne mort» - un coup d'œil sur la table des matières de ce numéro de Frontières suffira à nous convaincre de l'ampleur et de la diversité des perspectives à explorer. Quelle que soit notre position de départ dans ce débat de société sur les enjeux de fin de vie, nous aurons de meilleures chances d'atteindre notre visée - la vie bonne jusqu'à la mort - si nous acceptons de reconnaître que nous ne savons pas nécessairement déjà, au moment d'amorcer ces échanges entre citoyens, tout ce que nous aurions intérêt à savoir pour comprendre vraiment et contribuer utilement.

Gravement malades ou au bout de notre âge, nous allons tout perdre, ai-je dit? Peut-être pas... Nous conserverons l'actif et le passif du citoyen que nous sommes, dans les «institutions justes » de la société que nous habitons et que nous aurons aidé à construire, débat après débat, vote après vote. 


\section{Bibliographie}

ASSOCIATION CANADIENNE DE SOINS PALLIATIFS (2006). Document de réflexion de l'ACSP sur l'euthanasie, le suicide assisté et les soins de fin de vie de qualité, approuvé le 22 novembre, 6 p.

DUNSMUIR, M. et M. TIEDEMANN (2006). «L'euthanasie et l'aide au suicide au Canada », Bulletin, no 91-9F, février 1992 avec mises à jour périodiques, révisé le 23 février 2006, Gouvernement du Canada, Division du droit et du gouvernement, Sénat canadien, 21 p.

HANES, ALLISON (2006). "A mother and her son's final wish", The Gazette, Montréal, le vendredi 27 janvier 2006, p. A8.

OBSERVATOIRE DE L'ADMINISTRATION PUBLIQUE DE L'ÉNAP (2005). "Les droits reconnus aux malades en fin de vie», Le Soleil, Québec, le 7 février, p. A15.

PLATON (1950). «Les Lois », dans CEuvres complètes, tome II, Paris, Gallimard, La Pléiade.

RÉSEAU DE SOINS PALLIATIFS DU QUÉBEC (2007). Plan stratégique 2007-2010, 9 février, $14 \mathrm{p}$.

RICOEUR, P. (1995). Soi-même comme un autre, Paris, Seuil, 425 p. SÉNAT CANADIEN (1995). De la vie et de la mort-Rapport final, Comité spécial sur l'euthanasie et l'aide au suicide, juin, 28 p.

\section{Notes}

1. Au Québec, les principes relatifs à l'intégrité des personnes sont énoncés dans les articles 10 à 24 du premier chapitre du Code civil du Québec; la dispensation des soins nécessite le consentement libre et éclairé de la personne concernée. Le patient majeur et apte peut refuser les soins qu'on lui propose, même ceux qui sont indispensables pour le garder en vie.

2. Le témoignage de Marité Villeneuve, dans ce numéro, à la page 93, est particulièrement éloquent à cet égard.

3. Pour le Canada et le Québec, voir les données et statistiques citées dans ce numéro par Décarie et al., p. 64, ainsi que Saint-Arnaud et al., p. 87. Voir aussi l'entrevue avec $\mathrm{D}^{\mathrm{r}} \mathrm{A}$. Towers, dans Frontières, vol. $18, \mathrm{n}^{\mathrm{o}} 2$, p. $46-50$.

4. Pour la description d'un projet novateur mis de l'avant par un Centre de santé et de services sociaux du Québec afin de faciliter la communication en temps opportun des volontés du malade au sujet de l'intensité des soins qu'il reçoit, voit Shidler et Léger, dans ce numéro, p. 81.

5. Au Canada, les définitions les plus souvent citées sont celles qui figurent dans le Rapport publié en 1995 par le Sénat. Euthanasie: «un acte qui consiste à provoquer intentionnellement la mort d'autrui pour mettre fin à ses souffrances ». Aide au suicide: «le fait d'aider quelqu'un à se donner volontairement la mort en lui fournissant les renseignements ou les moyens nécessaires, ou les deux. [...] Pour qu'il y ait aide au suicide, il faut que le patient soit l'agent de sa mort, mais que celle-ci résulte de l'assistance d'un tiers ».

6. Pour la situation en Belgique voir, dans ce numéro, Bilsen et al., p. 76, et Schiffino et Bert, p. 40.

7. On peut lire, entre autres, l'article écrit par Allison Hanes (2006) dans le journal The Gazette; on y trouve aussi, dans un encadré, la version en anglais de la lettre écrite à Marielle Houle par son fils Charles, la remerciant d'avoir accepté de l'aider à mettre fin à ses souffrances.

8. Voir le texte de Lamarche, dans ce numéro, p. 97 sur la création de l'Association québécoise pour le droit de mourir dans la dignité (AQDMD).

9. Voir les remarques de Daneault, dans ce numéro p. 32, sur l'amicus mortis, l'ami qui aide le mourant. On peut penser que la génération qui grandit présentement en lisant la saga de Harry Potter trouvera une application particulièrement frappante de ce concept dans le dernier livre de la série, Les reliques de la mort, où le lecteur découvre qu'un personnage majeur, le magicien Dumbledore, sachant qu'il ne lui restait que quelques mois à vivre, avait demandé à un collègue, le professeur Rogue, de le tuer lui-même plutôt que de le laisser tomber entre les mains de ses ennemis, qui lui auraient réservé une mort lente et douloureuse.

10. Il est intéressant de lire, en parallèle avec cet extrait de Platon, la conversation entre deux médecins et un patient en fin de vie citée par Ollivier, dans ce numéro, p. 38.

11. Le texte de ce projet de loi est accessible en ligne: <www2.parl. gc.ca/HousePublications/Publication.aspx?pub=bill\&doc= $\mathrm{C}-407 \&$ parl $=38 \&$ ses $=1 \&$ Language $=\mathrm{F}>$.

Ce projet de loi a donné lieu à une heure de débats à la Chambre des communes le 31 octobre 2005 (la transcription des débats est accessible en ligne: $<w w w 2 . p a r l . g c . c a / H o u s e P u b l i c a t i o n s / P u b l i c a t i o n . a s p x ?$ Language $=\mathrm{F} \& \mathrm{Pub}=$ Hansard $\&$ Mode $=1 \& \operatorname{Parl}=38 \& \mathrm{Ses}=1 \& \mathrm{Doc}=144 \#$ Int-1443235>). Le projet de loi C-407 devait faire l'objet d'un vote en décembre 2005, mais ce vote n'a pas eu lieu en raison de la dissolution du Parlement et de la convocation d'une élection fédérale. La députée Francine Lalonde a indiqué son intention de déposer un nouveau projet de loi sur le même sujet.

12. Ce document en date du 9 février 2007 est accessible en ligne : <www. acsp.net/politique_publique/issues_sma/SMA-document_de_reflexionACSP-22nov2006.pdf $>$.

13. Ce document est accessible en ligne sur le site du Réseau : $<$ reseaupalliatif. org/images/PlanStrategique_2007-2010.pdf>.

\section{FRONTIÈRES PRÉSENTE DANS CE NUMÉRO DES GUVRES DE LUCE LAMOUREUX}

Luce Lamoureux, aquarelliste depuis plus de vingt ans, s’inspire de la flore pour créer un univers de poésie.

Elle a réalisé plusieurs expositions solo depuis 1994 et remporté plusieurs prix et mentions lors de divers événements. Ses aquarelles ont fait l'objet d'un article dans l'«Art au féminin» de l'édition du printemps 2003 de Magazin'Art. Ses œuvres figurent dans les répertoires d'artistes comme le biennal Magazin'Art et le Guide de Roussan depuis plusieurs années.

On peut retrouver ses œuvres dans plusieurs collections et dans différentes galeries comme à la Galerie Symbole Art à la Place Normandie et chez Arte Bella sur la rue Crescent, à Montréal, On la retrouve aussi à la Galerie Artazo à Sherbrooke, à la Galerie Courtemanche à Magog, à la Galerie L'Événement à Valleyfield et les Imagiers à Sutton. 